PDES, SUBMANIFOLDS AND

AFFINE DIFFERENTIAL GEOMETRY

BANACH CENTER PUBLICATIONS, VOLUME 69

INSTITUTE OF MATHEMATICS

POLISH ACADEMY OF SCIENCES

WARSZAWA 2005

\title{
SOME REMARKS ON THE KOZLOWSKI-SIMON CONJECTURE FOR AFFINE OVALOIDS
}

\author{
ZEJUN HU \\ Department of Mathematics, Zhengzhou University \\ Zhengzhou 450052, People's Republic of China \\ E-mail:huzj@zzu.edu.cn \\ GUOSONG ZHAO \\ Department of Mathematics, Sichuan University \\ Chengdu 610064, People's Republic of China \\ E-mail:gszhao@scu.edu.cn
}

Dedicated to Professor Udo Simon on the occasion of his 65th birthday

\begin{abstract}
In this note, we are concerned with the Kozlowski-Simon conjecture on ovaloids and prove that it is correct under additional conditions.
\end{abstract}

1. Introduction. Let $A^{n+1}$ be the unimodular affine space of dimension $n+1$. A connected, compact, locally strongly convex hypersurface without boundary in $A^{n+1}$ is called an ovaloid. In 1923, W. Blaschke made the following conjecture (cf. [Bl]):

An ovaloid $x: M^{2} \rightarrow A^{3}$ with affine metric of constant scalar curvature must be an ellipsoid.

In [Sch] R. Schneider proved that if an ovaloid $M$ and an ellipsoid are equiaffinely isometric, that is, they have the same Blaschke metric, then $M$ is an ellipsoid. This result solves the above conjecture. Later, M. Kozlowski and U. Simon gave the following generalization for general dimensions (cf. [K-S] or [L-S-Z]):

2000 Mathematics Subject Classification: 53C21, 53C42, 53A15.

Key words and phrases: Kozlowski-Simon conjecture, locally conformally flat, harmonic metric, Blaschke hyperovaloid.

Z. J. Hu partially supported by grants of SRF for ROCS, NSFC and OYF of Henan, China. G. S. Zhao partially supported by DFG, NSFC and Qui Shi Award.

The paper is in final form and no version of it will be published elsewhere. 
TheOREM 1. Let $x: M^{n} \rightarrow A^{n+1}$ be an ovaloid of dimension $n$; if $R$ is constant for $n=2$, or $\left(M^{n}, G\right)$ is an Einstein space for $n \geq 3$, then $x(M)$ must be an ellipsoid. Here $G$ is the Blaschke metric and $R$ is the scalar curvature of $G$.

Based on the above theorem, M. Kozlowski and U. Simon made the following conjecture, which up to now is still open for $n \geq 3$.

Kozlowski-Simon Conjecture (see [K-S] and [L-S-Z]). Let $x: M^{n} \rightarrow A^{n+1}$ be an ovaloid. If the scalar curvature of $M$ is constant, then $x(M)$ is an ellipsoid.

Toward this conjecture, G. S. Zhao made another contribution by proving the following result.

Theorem $2([\mathrm{Zh}])$. Let $x: M^{n} \rightarrow A^{n+1}$ be an ovaloid of dimension $n$. If $\left(M^{n}, G\right)$ has a parallel Ricci curvature tensor, then it is locally the Riemannian product of some Einstein manifolds and consequently $x(M)$ must be an ellipsoid.

In this note, we will contribute to Kozlowski-Simon's problem by proving the following results.

TheOREm 3. Let $x: M^{n} \rightarrow A^{n+1}(n \geq 3)$ be an ovaloid and assume that the Blaschke metric $G$ of $x(M)$ is locally conformally flat and possesses constant scalar curvature. Then $x(M)$ must be an ellipsoid.

There is an analogous result for compact hypersurfaces in Euclidean space, see e.g. Theorem 4.2 in [Ch]:

TheOREM $([\mathrm{Ch}])$. An n-dimensional $n>3$ compact, connected orientable, locally conformally flat hypersurface in Euclidean space with constant scalar curvature is isometric to a standard sphere.

We would like to point out that our method of proof can also be used to prove Cheng's theorem.

Theorem 4. Let $x: M^{3} \rightarrow A^{4}$ be an ovaloid; if the Blaschke metric $G$ of $x(M)$ is of harmonic curvature, then $x(M)$ is an ellipsoid.

\section{Some results on locally conformally flat manifolds and manifolds with} harmonic curvature. Recall that an $n$-dimensional Riemannian manifold $\left(M^{n}, g\right)$ is said to be locally conformally flat if it admits a coordinate covering $\left\{U_{\alpha}, \varphi_{\alpha}\right\}$ such that the map $f_{\alpha}:\left(U_{\alpha}, g_{\alpha}\right) \rightarrow\left(S^{n}, g_{0}\right)$ is a conformal map, where $g_{0}$ is the standard metric on $S^{n}$.

Firstly, we recall the following well-known results due to N. H. Kuiper and M. Obata, respectively.

LEMma 1 (cf. $[\mathrm{K}])$. A conformally flat, simply connected Riemannian manifold $\left(M^{n}, g\right)$ is conformorphic to an open submanifold of $S^{n}(1)$. In particular, if $M^{n}$ is compact, then $\left(M^{n}, g\right)$ is conformorphic to $S^{n}(1)$.

LEMma 2 (cf. [O]). Let $\left(S^{n}(1), g\right)$ be the unit sphere with $g$ the standard metric, if $\tilde{g}$ is another Riemannian metric on $S^{n}$ and if $\tilde{g}$ is conformal to $g$ and is of constant scalar curvature, then $\tilde{g}$ must have constant sectional curvature. 
Secondly, recall that a Riemannian manifold $\left(M^{n}, g\right)$ is said to have harmonic curvature if its curvature tensor satisfies $\delta R=0$, i.e., $\nabla^{i} R_{h i j k}=0$. (For details on this notion, see e.g. [D1, D2]). If $\left(M^{n}, g\right)$ is of harmonic curvature, then by using the second Bianchi identity, we have

$$
\nabla^{i} R_{h i j k}=\nabla_{k} R_{h j}-\nabla_{j} R_{h k}=0 .
$$

By contracting the indices $h, k$, we have

$$
-\nabla^{i} R_{i j}=\nabla^{i} R_{i j}-\nabla_{j} R=0 .
$$

Thus $\nabla_{j} R=0$ and $\left(M^{n}, g\right)$ is of constant scalar curvature. Thus we obviously have

Lemma 3. Every Riemannian manifold with parallel Ricci tensor is of harmonic curvature and every Riemannian manifold with harmonic curvature is of constant scalar curvature.

Of course, the condition of constant scalar curvature is less restrictive than that of harmonic curvature. Recalling Theorem 1 and Theorem 2, we hope that one might be able to prove that both conditions are equivalent for Blaschke hyperovaloids.

REMARK 1. In general, the conditions on harmonic curvature and having that of parallel Ricci tensor are quite different. In fact, A. Derdzinski obtained the following results in $[\mathrm{D} 1, \mathrm{D} 2]$ :

(i) For each $n \geq 3$, there exist Riemannian manifolds $\left(M^{n}, g\right)$ having harmonic curvature whereas the Ricci tensor of $g$ is not parallel, and consequently $\left(M^{n}, g\right)$ is not Einstein. Such examples exist for both $M^{n}$ being compact and non-compact.

(ii) If $M^{n}$ is compact, all known examples in (i) are manifolds $M^{n}$ that are not simply connected.

\section{Proof and remark of the results}

Proof of Theorem 3. If $\left(M^{n}, G\right)$ is conformally flat and has constant scalar curvature, then from the fact that $M^{n}$ is compact and simply connected and by Lemma 1 and Lemma $2,\left(M^{n}, G\right)$ must be conformorphic to $\left(S^{n}(1), g\right)$ and have constant sectional curvature. From Theorem 1, $x(M)$ must be an ellipsoid.

REMARK 2. The main interest in Theorem 3 is that the conformal flatness is an assumption on the class of relative metrics, not specifically on the Blaschke metric. Thus this result leads to another class of problems: Consider a hyperovaloid with a conformally flat class of relative metrics. Under which further assumptions, depending only on the class of relative geometries and not on a specific relative geometry, can one characterize hyperellipsoids? That one needs further assumptions besides conformal flatness is clear from known results: For a non-degenerate hypersurface of revolution the class of relative metrics is conformally flat. For related results we refer to $[\mathrm{B}-\mathrm{K}]$ and $[\mathrm{S}]$.

Proof of Theorem 4. $\left(M^{3}, G\right)$ has harmonic curvature, so we have $\nabla_{i} R_{j k}=\nabla_{j} R_{i k}$ and $R=$ const. 
We note that a 3-dimensional Riemannian manifold is conformally flat if and only if the Schouten tensor satisfies

$$
C_{i j k}:=R_{i j, k}-R_{i k, j}+\frac{1}{4}\left(g_{i k} R_{, j}-g_{i j} R_{, k}\right)=0 .
$$

Now, we can see that harmonic curvature implies that $\left(M^{3}, G\right)$ is conformally flat. Then the conclusion follows from Theorem 3 .

In $[\mathrm{D}-\mathrm{G}]$, the authors proved the following

THEOREM 5. Let $M$ be an n-dimensional, compact, connected and nonnegatively curved Riemannian manifold of constant scalar curvature. If the Ricci tensor of $M$ satisfies

$$
(\nabla \operatorname{Ric})(X, Y, Z)=(\nabla \operatorname{Ric})(Y, X, Z), \quad X, Y, Z \in \Gamma(T M)
$$

and $I^{0}(M) \neq C^{0}(M)$, then $M$ is isometric to $S^{n}$. Here $I^{0}(M)$ and $C^{0}(M)$ are the identity components of the isometry group $I(M)$ of $M$ and the group of conformal transformations $C(M)$ of $M$, respectively.

From the proof of Theorem 5 , we know that if $\left(M^{n}, g\right)$ is a compact, connected and nonnegatively curved Riemannian manifold of constant scalar curvature, then the condition of harmonic curvature is equivalent to the condition that the Ricci curvature tensor is parallel. Combining this fact with Theorem 2, we obtain immediately the following

TheOREM 6. Let $x: M^{n} \rightarrow A^{n+1}(n \geq 3)$ be an ovaloid and assume that the Blaschke metric $G$ of $x(M)$ is harmonic and has nonnegative sectional curvature. Then $x(M)$ must be an ellipsoid.

Finally, one can deduce immediately from the proof of Theorem 4 the following

THEOREM 7. Let $M^{3}$ be compact and have finite fundamental group, then a Riemannian metric $g$ on $M^{3}$ is harmonic if and only if it has constant sectional curvature.

Proof. Assume that $\left(M^{3}, g\right)$ is harmonic. Let $\tilde{M}^{3}$ be the universal covering of $M^{3}$, since $M$ has finite fundamental group, $\tilde{M}^{3}$ is compact. Denote by $\tilde{g}$ the lifting of $g$ in $\tilde{M}$. Then $(\tilde{M}, \tilde{g})$ is locally isometric to $\left(M^{3}, g\right)$ and has harmonic curvature, thus $\tilde{g}$ has constant sectional curvature by the same argument as in the proof of Theorem 4 . Therefore, $g$ has constant sectional curvature. The converse is trivial.

Remark 3. As a comparison with Theorem 7, we note that A. Derdzinski had constructed many compact examples $\left(M^{3}, g\right)$ with the properties that $\delta R=0$ but $\nabla_{k} R_{i j} \neq 0$, in all his examples, $M^{3}$ has infinite fundamental group and is diffeomorphic to $S^{1} \times N$ and $N$ is a compact and simply connected manifold.

Acknowledgements. The authors would like to thank the referee for both helpful comments and informing them about the papers $[\mathrm{B}-\mathrm{K}]$ and $[\mathrm{S}]$.

\section{References}

[Bl] W. Blaschke, Vorlesungen über Differentialgeometrie, II, Berlin, J. Springer, 1923. 
[B-K] M. Becker and W. Kühnel, Hypersurfaces with constant inner curvature of the second fundamental form, and the non-rigidity of the sphere, Math. Z. 223 (1996), 693-708.

[Ch] Q.-M. Cheng, Topology and geometry of complete submanifolds in Euclidean spaces, this volume, 67-80.

[D1] A. Derdzinski, Classification of certain compact Riemannian manifolds with harmonic curvature and non-parallel Ricci tensor, Math. Z. 172 (1980), 273-280.

[D2] A. Derdzinski, On compact Riemannian manifolds with harmonic curvature, Math. Ann. 259 (1982), 145-152.

[D-G] S. Deshmukh and M. A. Al-Gwaiz, Compact manifolds of constant scalar curvature, Geometriae Dedicata 40 (1991), 23-27.

[K] N. H. Kuiper, On conformal flat spaces in the large, Ann. Math. 50 (1949), 916-924.

[K-S] M. Kozlowski and U. Simon, Hyperflächen mit äquiaffiner Einsteinmetrik, in: Mathematica Festschrift E. Mohr, TU Berlin, 1985, 179-190.

[L-S-Z] A.-M. Li, U. Simon and G. S. Zhao, Global Affine Differential Geometry of Hypersurfaces, W. de Gruyter, Berlin, New York, 1993.

[O] M. Obata, The conjecture on conformal transformations of compact Riemannian manifolds, J. Diff. Geom. 6 (1971), 247-258.

[S] C. Scharlach, Affin-konforme Geometrie regulärer Hyperflächen, Diploma Thesis, FB Mathematik, TU Berlin 1989.

[Sch] Z. Schneider, Zur affinen Differentialgeometrie im Grossen I, Math. Z. 101 (1967), 375-406.

[Zh] G. S. Zhao, Ovaloids with parallel Ricci curvature tensor, J. of Sichuan University, Natural Science Edition, 35 (1998), 174-177. 